Metzler Lexikon der deutsch-jüdischen Literatur 


\section{Metzler Lexikon der deutsch-jüdischen Literatur}

Jüdische Autorinnen und Autoren deutscher Sprache von der Aufklärung bis zur Gegenwart Mit 255 Abbildungen

Herausgegeben von Andreas B. Kilcher

Verlag J. B. Metzler

Stuttgart $\cdot$ Weimar 


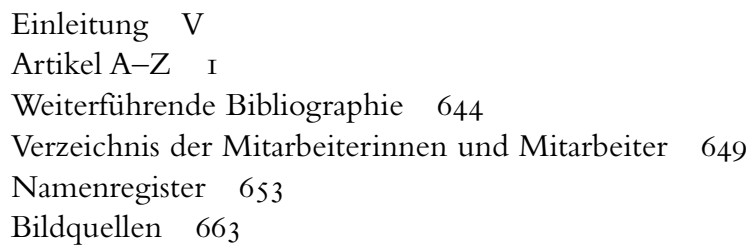

Die Deutsche Bibliothek - CIP-Einheitsaufnahme

Metzler-Lexikon der deutsch-jüdischen Literatur : jüdische Autorinnen und Autoren deutscher Sprache von der Aufklärung bis zur Gegenwart / hrsg. von Andreas B. Kilcher. - Stuttgart ;

Weimar : Metzler, 2000

ISBN 978-3-476-0I682-9

ISBN 978-3-476-03782-4 (eBook)

DOI I0.1007/978-3-476-03782-4

Dieses Werk einschließlich aller seiner Teile ist urheberrechtlich geschützt. Jede Verwertung außerhalb der engen Grenzen des Urheberrechtsgesetzes ist ohne Zustimmung des Verlages unzulässig und strafbar. Das gilt insbesondere für Vervielfältigungen, Übersetzungen, Mikroverfilmungen und die Einspeicherung und Verarbeitung in elektronischen Systemen.

(C) 2000 Springer-Verlag GmbH Deutschland

Ursprünglich erschienen bei J. B. Metzlersche Verlagsbuchhandlung und Carl Ernst Poeschel Verlag GmbH in Stuttgart 2000 


\section{Einleitung}

Als Ludwig Geiger, einer der Wortführer des deutschen liberalen Judentums, vor rund hundert Jahren als einer der ersten Literaturwissenschaftler die Frage nach der deutsch-jüdischen Literatur stellte, begründete er dies mit einem in mancher Hinsicht zukunftsweisenden Verständnis der deutschen Literatur, der Literatur überhaupt. Gegen das im I9. Jahrhundert vorherrschende Konzept einer >Nationalliteratur und damit auch gegen eine dezidiert sdeutsche Literatur entwickelte er in seinen 1903/04 in Berlin gehaltenen Vorträgen unter dem Titel Die Juden und die deutsche Literatur das Programm einer Literatur, die kulturelle und nationale Grenzen überschreitet. Insbesondere die deutsche Literatur wollte er als ein kosmopolitisches und interkulturelles System verstanden wissen: „Wer die deutsche Literatur und Kunst [...] betrachtet, der wird geradezu sagen müssen, daß es eine ausschließlich deutsche Kunst fast niemals gegeben hat. «" Dem verlieh einige Jahre später, nunmehr vor dem Hintergrund des Zionismus, der Journalist und Verleger Gustav Krojanker Nachdruck, dessen vielbeachtete Aufsatzsammlung Die Juden in der deutschen Literatur (1922) zusammen mit Geigers Schrift zu den wichtigen Beiträgen jüdischer Intellektueller zur Debatte über die deutsch-jüdische Literatur zählt. Mit Geiger hält Krojanker gegen einen puristischen literarischen »Normalbegriff, der zwischen deutsch und undeutsch leichthin scheidet", einen "weiteren und wahreren«, nämlich einen "Europa«-Begriff von Literatur. Auch Krojanker gilt gerade die deutsche Literatur "als Inbegriff recht verschiedenartiger Prägungen ${ }^{2}$ kultureller Systeme. So unterschiedlich Geiger und Krojanker die deutsch-jüdische Literatur letztlich auch verstehen - Geiger argumentiert mehr von der deutschen, Krojanker mehr von der jüdischen Kultur her -, stimmen doch beide darin überein, daß sie die deutsche Literatur, Literaturen überhaupt, nicht als monokulturelle und nationale Phänomene erachten, sondern als plurale diskursive Felder, auf denen sich die semiotischen Systeme verschiedener Kulturen überkreuzen und verbinden - ein interkulturelles Dispositiv, das sie mit dem Namen "Europa" versehen haben. Insbesondere die deutsch-jüdische Literatur gilt ihnen als das plausibelste Beispiel dieser interkulturellen Konstitution von Literatur: Sie ist weder "jüdisch" noch "deutsch", sondern beides - in seiner jeweiligen Vielfalt - zugleich und damit Paradigma einer kosmopolitischen, »europäischen« Literatur.

Dieses geradezu zukunftsweisende Verständnis der deutsch-jüdischen Literatur war jedoch zur Zeit seiner Formulierung um I900 kaum die Beschreibung einer Realität; es hatte vielmehr den Charakter eines - auf unterschiedliche Weise verstandenen - Postulats, das bewußt gegen die Bedingungen einer weitaus disharmonischeren historischen Wirklichkeit gestellt wurde. Tatsächlich war das Feld der deutsch-jüdischen Literatur nur selten der Ort eines symbiotischen Austauschs, wofür als beispielhaft, nach der wegweisenden Freundschaft zwischen Moses Mendelssohn und Gotthold Ephraim Lessing, vielleicht der Kreis jüdischer Schriftsteller

I Ludwig Geiger, Die deutsche Literatur und die Juden, Berlin I9I0, 5.

2 Gustav Krojanker, Die Juden in der deutschen Literatur, Berlin I922, Io. 
und Intellektueller um Stefan George - Karl Wolfskehl allen voran - gelten könnte. In weiten Teilen aber war die deutsch-jüdische Literatur ein Schauplatz von Ausgrenzung, Verletzung und Gewalt. Genau in diesem Sinne hatte beispielsweise Joseph Roth - angesichts der Bücherverbrennung von I933, Ausdruck der auf die Kultur überspringenden Gewalt - verzweifelt und deshalb radikal von den deutschjüdischen Schriftstellern das Festhalten an einer europäischen deutschsprachigen Literatur eingefordert: "Wir deutschen Schriftsteller jüdischer Abstammung«, so Roth angesichts des Äußersten, "bleiben die Nachkommen der alten Juden, der Ahnen der europäischen Kultur, die einzig legitimen deutschen Repräsentanten dieser Kultur. $\aleph^{3}$ Nach 1945 hat George Steiner in seiner Analyse des "Unmenschlichen" - entschiedener noch - den "mitteleuropäischen Humanismus" letztlich als einen genuin jüdischen Wert verstanden, deren Anwalt zu sein den deutschjüdischen Schriftstellern überantwortet war, deutsch-jüdische Literatur also als kritische Instanz der Moral und des Humanismus im Jahrhundert ihrer Toterklärung. "Sogar das Wort >Mensch«", so Steiner, »hat für mich einen klaren deutschjüdischen Klang. $\star^{4}$

Spätestens hier wird deutlich, daß die aktuelle Rede von der deutsch-jüdischen Literatur keineswegs selbstverständlich, sondern beträchtlichen Schwierigkeiten ausgesetzt ist, die bei einem Vorhaben wie dem vorliegenden Lexikon von Anfang an offengelegt werden müssen. Sie manifestieren sich deutlich darin, daß der Begriff der "deutsch-jüdischen Literatur" seit seiner Erfindung im letzten Drittel des I9. Jahrhunderts - im Reflex einer mehr oder weniger physischen oder kulturellen Gewalt - lange Zeit polemisch, ideologisch und apologetisch verwendet wurde, kaum aber als eine wie auch immer "wertfreie» ästhetische und literaturtheoretische Kategorie. In der Rede von der deutsch-jüdischen Literatur überkreuzen sich auf eine für das Phänomen der Literatur ungewohnte und für neuere literaturtheoretische Positionen irritierende Weise ästhetische und ethische Kriterien und Argumente. Eine grundsätzliche Klärung des Begriffs ist deshalb am Eingang eines Vorhabens unabdinglich, das darin besteht, diesen interkulturellen Bereich als einen eigenen, wenn auch hochgradig heterogenen Diskurs im Schnittpunkt zwischen der deutschen und der jüdischen Kultur zu beschreiben. Diese Begriffsklärung besteht genauer darin, die kontroversen historischen Interpretationen des Konzeptes der deutsch-jüdischen Literatur im Kontext der verschiedenen Kommunikationszusammenhänge, in denen ihm auf unterschiedliche Weise Bedeutung und Funktion zugesprochen wurde, zu rekonstruieren. Kein literaturgeschichtlicher Überblick über die deutsch-jüdische Literatur also soll hier vorangestellt werden, sondern vielmehr eine begriffsgeschichtliche Klärung, die einer historischen Darstellung vorangehen muß. Denn erst mit diesem Wissen und Bewußtsein der kontroversen historischen Interpretationen der Gestalt und Funktion einer jüdischen Literatur und Kultur innerhalb der deutschen wird es - auch, aber nicht nur im Hinblick auf

3 Joseph Roth, Autodafé des Geistes, in: Werke, Bd. 3, hg. Klaus Westermann, Köln I989, $494 \mathrm{ff}$.

4 George Steiner, Sprache und Schweigen. Essays über Sprache, Literatur und das Unmenschliche, Frankfurt a. M. I973, 8. 
das vorliegende Lexikon - möglich sein, Parameter einer aktuellen und nichtideologischen Rede von der deutsch-jüdischen Literatur zu formulieren.

Die Erfindung des Begriffs der deutsch-jüdischen Literatur fällt ins letzte Drittel des I9. Jahrhunderts. ${ }^{5}$ Von der deutsch-jüdischen Literatur kann allerdings von Anfang an nicht die Rede sein. Denn schon um I90o lassen sich zumindest drei grundlegend verschiedene Konzeptionen unterscheiden, die teilweise unabhängig voneinander stehen, vielfach aber aufeinander Bezug nehmen, mehr noch: miteinander konkurrieren. Es waren dies allerdings drei Interpretationen der deutsch-jüdischen Literatur, die auch im Dienst politischer und kultureller Wertesysteme standen. Insofern sie Instrumente konkurrierender politisch-kultureller Diskurse waren, wird man sie, freilich auf ganz unterschiedliche Weise, als ideologisch verstehen müssen: erstens die deutsch-jüdische Literatur der völkischen Germanistik, zweitens die deutsch-jüdische Literatur des Kulturzionismus, drittens die deutsch-jüdische Literatur der Assimilation.

Was den Begriff der deutsch-jüdischen Literatur immer schon unter Legitimationsdruck gestellt hat, war seine $\mathrm{kra}$ polemisch-ideologische Verwendung innerhalb der völkischen Germanistik von ca. I87I bis 1945. Spätestens seit Eugen Dührings Die Judenfrage als Racen-, Sitten- und Culturfrage (I88I) gab es in Deutschland einen dezidiert antisemitischen Literaturbegriff. Sein Programm bestand darin, die deutsche Literatur von allem Naturalismus, Ästhetizismus, Modernismus, und das hieß zumeist, von den "verderblichen Eigenschaften« der »Judenrace« zu "reinigen". "Keiner Nation ist es", so klagte Dühring auch in Die Größen der modernen Literatur (I 893) über "das literarische Auftauchen des Hebräertums", "so handgreiflich übel gegangen, wie [...] den Deutschen. In dem Maße wie die ihrige wurde keine der europäischen Literaturen von Judennichtigkeiten absorbiert. " ${ }^{6}$ Mit anderen Worten: Dühring machte die rassische Ideologie zum Programm der deutschen Literaturwissenschaft: "Die Abwehr dieser antiarischen Verschlechterungsmache sollte allerdings im Antisemitismus einen Stützpunkt finden; aber dieser muß sich praktisch und äußerlich noch erst in das Literaturgebiet hinein verpflanzen. «7 Das von Dühring geforderte Programm hat am konsequentesten der Germanist Adolf Bartels umgesetzt. Sein Vorhaben einer "antisemitischen Literaturgeschichtsschreibung ${ }^{8}$ formulierte er in polemischen Schriften wie Das Judentum in der deutschen Literatur, erschienen als Anhang zu Kritiker und Kritikaster (1903), in dem Aufsatz Judentum und deutsche Literatur (1910) und am ausführlichsten in Jüdische Herkunft und Literaturwissenschaft (I925). Bartels' Ideologie ist ebenso simpel wie grausam: Sie

5 Zur grundsätzlichen Diskussion des Begriffs der deutsch-jüdischen Literatur vgl. Hana Wirth-Nesher (Hg.), What is Jewish Literature? Philadelphia I994; Hans Otto Horch, Was heißt und zu welchem Ende studiert man deutsch-jüdische Literaturgeschichte? Prolegomena zu einem Forschungsprojekt, in: German Life and Letters 49 (1996), I24-I35; Andreas B. Kilcher, Was ist deutsch-jüdische Literatur? Eine historische Diskursanalyse, in: Weimarer Beiträge I44 (I999), 485-5I7. Für weitere Angaben vgl. die Bibliographie.

6 Eugen Dühring, Die Größen der modernen Literatur, 2 Bde., Leipzig I893, Bd. 2, 277.

7 Ebd., 389.

8 Adolf Bartels, Jüdische Herkunft und Literaturwissenschaft, Leipzig I925, Io. 
beruht auf der These, daß Kulturen und Literaturen rassisch, ethnisch und national bedingte Systeme sind. Deshalb bestehe die eigentliche Aufgabe der deutschen Literaturwissenschaft in der wreinlichen Scheidung von Juden und Deutschen, Deutschtum und Judentum «": "Der Literaturhistoriker hat zu zeigen, was die unter uns lebenden produzierenden Juden von den Deutschen aufnehmen, wie sie das gemäß ihrem Rassencharakter verändern, was sie eigenes geben und wie sie durch das so entstandene Produkt auf das deutsche Volk wirken. ${ }^{\text {10 }}$

Wer auch immer sich mit der deutsch-jüdischen Literatur beschäftigt, sieht sich gezwungen, von dieser gewaltbereiten Ideologisierung sich abzugrenzen. Dies tat der jüdische Journalist und Schriftsteller Moritz Goldstein bereits I9I2, als er sich in einem vielbeachteten Aufsatz daran machte, den Deutsch-jüdischen Parnaß, den >Musenberg der jüdischen Literatur im Gebirge der deutschen Literaturlandschaft, auszumessen: "Die Juden in der deutschen Literatur', das ist eines von den heikelsten Dingen, die nicht in den Mund genommen werden dürfen, will man sich nicht heillos kompromittieren. " ${ }^{\text {I }}$ Auch Gustav Krojanker verwies, als er zehn Jahre später seine Aufsatzsammlung Juden in der deutschen Literatur vorlegte, auf dieses Problem: "Das Unternehmen dieses Buches, den Juden innerhalb des deutschen Kulturkreises als eine Sondererscheinung zu sehen; mehr noch: diesen Gesichtspunkt in den geheiligten Bezirk der Literatur zu tragen, wo, wenn irgendwo, der gute Europäer gilt - es ist ein ungemein verdächtiges Unternehmen. Denn es scheint in diesem Deutschland fast nicht anders denkbar, als daß die Geschäfte einer finsteren Reaktion betreibt, wer das Wesen des Juden als ein unterschiedliches überhaupt nur zu betrachten wagt. [...] Kurz: das Problem des jüdischen Menschen im deutschen Kulturkreis, die Frage nach dem Unterschiedlichen seines Wesens ist bis heute vorwiegend dem Antisemitismus überlassen worden. ${ }^{\mathrm{I2}}$ Dieselbe Schwierigkeit gewann besonders nach 1945 an Gewicht. Angesichts dessen hat vor kurzem noch Ernst Gombrich in seiner "Diskussionsbemerkung" Jüdische Identität und jüdisches Schicksal (I997) jede noch so sgut gemeinter Rede von einer deutsch-jüdischen Kultur in moralischer und politischer Hinsicht als illegitim und geradezu gefährlich zurückgewiesen. ${ }^{13}$ Wer von deutsch-jüdischer Kultur spreche, begebe sich automatisch auf das Argumentationsniveau des Nationalsozialismus und der Nürnberger Gesetze. Gemäß Gombrich tradiert der Begriff der deutsch-jüdischen Literatur unweigerlich die nazistische Ideologie einer jüdischen Sonderliteratur mit allen gewaltsamen politischen Implikationen der Ausgrenzung.

Gewiß muß jede aktuelle Rede von der deutsch-jüdischen Literatur gerade diese Art der Ideologisierung ganz bewußt zurückweisen, und zwar noch heute - nicht etwa, weil sie in ihren Argumenten ernst genommen werden müsste, sondern vor allem zu Händen eines historischen Bewußtseins. Doch wäre es wiederum geradezu

9 Bartels, Jüdische Herkunft, I I.

Io Bartels, Judentum und deutsche Literatur, Berlin I9Io, 5.

I I Moritz Goldstein, Deutsch-jüdischer Parnaß, in: Der Kunstwart 25 (März I9I2), 28I-294, hier: $28 \mathrm{I}$.

I2 Krojanker, Juden in der deutschen Literatur, 7.

I3 Ernst Gombrich, Jüdische Identität und jüdisches Schicksal. Eine Diskussionsbemerkung, Wien I997. 
unhistorisch, den Begriff der deutsch-jüdischen Literatur - und damit letztlich jede Rede über das Judentum - auf die völkische Germanistik und die Nürnberger Gesetze zu reduzieren. Denn die deutschsprachige Literatur jüdischer Autoren war auch und vor allem Gegenstand einer breiten und vielstimmigen innerjüdischen Debatte. Dabei standen sich insbesondere die Positionen des Kulturzionismus auf der einen und diejenige der Assimilation auf der anderen Seite gegenüber. Beide Positionen haben auf je eigene Weise eine ideologische Komponente, insofern sie im Kontext zweier kulturpolitischer Programme standen, die dem jüdischen Selbstbestimmungsdiskurs der Moderne angehören. Es waren dies zwei Interpretationen der deutsch-jüdischen Literatur, denen es auf unterschiedliche Weise darum ging, teilweise auch gegen das völkische "Argument, den Juden das Schöpferische abzusprechen ${ }^{14}$, die kulturelle Produktivität der Juden hervorzuheben, sei es mit dem Ziel der Etablierung einer eigenständigen jüdischen Literatur wie im Kulturzionismus, sei es, gemäß der Akkulturationstheorie, mit dem Ziel der Hervorhebung und Integration des "jüdischen Beitrags" zur deutschen Kultur.

Den kulturzionistischen Interpretationen kam es darauf an - die völkische Politik der Sonderung unter gegenteilige Vorzeichen setzend -, "das Jüdische" in der deutschen Literatur hervorzuheben und letztlich zu zeigen, daß die Texte deutschsprachiger Autoren jüdischer Herkunft nicht in das Kontinuum der deutschen, sondern in dasjenige der jüdischen Literatur gehören. Dabei gibt es keine einheitliche Vorstellung über die jüdische Identität der deutschsprachigen Literatur jüdischer Autoren, sondern vielmehr eine Vielzahl differierender Interpretationen, von denen zumindest zwei deutlich umrissene Positionen genannt seien. Gemäß einer konsequenteren zionistischen Kulturtheorie wird die jüdische Identität der Literatur in erster Linie durch die hebräische Sprache definiert, eine deutschsprachig-jüdische Literatur deshalb letztlich als unmöglich erachtet. Gemäß eines weitergefaßten und liberaleren Kulturzionismus wird die jüdische Identität vornehmlich auf der Inhaltsseite von Literatur verortet und damit die Bestimmung eines "jüdischen Stoffes" oder "Geistes" konstitutiv für die deutsch-jüdische Literatur. In diesem Sinne unterschied beispielsweise Moritz Goldstein in seinem genannten Aufsatz und erneut in der kurz danach verfaßten Schrift Begriff und Programm einer jüdischen Nationalliteratur (I9I3) zwischen einer radikal zionistischen Literatur, die letztlich nur eine hebräische Literatur sein kann, und einer gemäßigt nationaljüdischen, deutschsprachig-jüdischen Literatur, die sich über Stoffe als "jüdisch" definiert. Der radikale Begriff grenzt die jüdische ganz aus der deutschen Literatur aus: "Ich komme zu der Erkenntnis, daß die geistige Organisation der deutschen Juden ein Traum ist und daß nur mit Hilfe der hebräischen Sprache und international eine jüdische Kultur zu finden ist. ${ }^{\mathrm{IS}}$ Der konsequente "Zionismus für die Kunst«, also die »Wiederbelebung hebräischer Sprache und Poesie«" ${ }^{16}$, erweist

I4 Vgl. die Debatte, die u.a. Julius Bab, Ludwig Strauß und Arnold Zweig in der Freistatt führten. Vgl. etwa Arnold Zweig, Zum Problem des jüdischen Dichters in Deutschland, in: Die Freistatt I, Nr. 5 (August 1913), 375-380.

I5 Moritz Goldstein, Texte zur jüdischen Selbstwahrnehmung, in: Aschkenas 7 (1997), 79-135, hier: I3I.

I6 Goldstein, Deutsch-jüdischer Parnaß, 290. 
sich für Goldstein selbst jedoch als ein Postulat. Für sein eigenes Schreiben wie für die "westeuropäische« Konstitution der deutsch-jüdischen Literatur überhaupt erachtet er den kulturzionistischen »Sprung in die neuhebräische Literatur" als letztlich nicht realisierbar: "Wir deutschen Juden [. . . können ebensowenig hebräische Dichter werden, wie wir nach Zion auswandern. $"{ }^{17}$ Deshalb fordert er eine dezidiert deutschsprachige jüdische "Nationalliteratur", die sich über die Sprache zwar als deutsch auszeichnet, über die Stoffe jedoch eine jüdische kulturelle Identität konstituiert. »Wir brauchen«, so Goldstein, »den Juden auch als Objekt der Dichtung«, "wir wollen das jüdische Heldenideal. ${ }^{18}$ Es ist nur konsequent, wenn in der nachfolgenden sogenannten »Kunstwart-Debatte« Goldsteins Position von zionistischer Seite, etwa von Martin Bubers Schwiegersohn Ludwig Strauß, zwar gelobt, aber dennoch der deutsch-jüdischen "Zwitterkultur» zugerechnet wurde, die es doch im »Übergang zur neuhebräischen Literatur» letztlich zu überwinden gelte. $^{19}$

Während das apologetische Argument des Kulturzionismus darin besteht, die deutschsprachige Literatur jüdischer Autoren vor allem vermittels des Kriteriums des Stoffs an das Kontinuum der jüdischen Literatur zurückzubinden, geht es in der Kulturtheorie der Assimilation gerade umgekehrt darum, die deutsch-jüdische Literatur, wenn auch mit dem Hinweis auf den bedeutenden jüdischen "Anteil und "Beitrag", vor allem vermittels des Kriteriums der deutschen Sprache in das Kontinuum der deutschen Literatur zu stellen. Träger dieser Vorstellung waren die bürgerlich-liberalen deutschen Juden, die sich in erster Linie als »deutsche Staatsbürger" verstanden und ihr Judentum einzig über den »jüdischen Glauben" definierten. Ludwig Geiger beispielsweise, auch als Herausgeber der Allgemeinen Zeitung des Judentums (1909-I9) prominenterVertreter dieser Position, richtete sich konsequenterweise nicht nur gegen den völkischen, sondern in einem Atemzug auch gegen den zionistischen Literaturbegriff, wenn er monierte, daß die deutsch-jüdische nicht auf jüdische Literatur reduziert werden dürfe, sondern vielmehr der deutschen Literatur angehöre. Der völkischen Germanistik wirft er vor, Literaturwissenschaft auf die biologistische Frage der "Herkunft" zu reduzieren und "Schriftsteller zu Juden zu stempeln « ${ }^{20}$ Dem zionistischen Literaturbegriff auf der anderen Seite wirft Geiger vor, im Dienst eines neuen jüdischen Selbstbewußtseins seinerseits einem kulturellen Purismus und Separatismus zu verfallen: "Nicht minder weise ich als völlig unwissenschaftlich alle Bemühungen und Anschauungen von mir, die, durch die Unzufriedenheit mit den augenblicklichen Verhältnissen, durch Kränkungen, die der einzelnen Person oder der Glaubensgemeinschaft zugefügt wurden, entstanden, ein jüdisches Stammesbewußtsein konstruieren und den Traum einer jüdischen Nationalität oder gar eines jüdischen Staates darstellen, oder sich direkt bemühen, ihn in die Wirklichkeit umzusetzen. Ich beklage die zahlreichen Romane, Gedichte und Dramen, die in neuerer Zeit von deutschen Juden veröffentlicht worden sind,

I7 Ebd., 290 f.

I 8 Goldstein, Begriff und Programm einer jüdischen Nationalliteratur, Berlin I9I3, I6ff.

I9 Franz Quentin [= Ludwig Strauß], Aussprache zur Judenfrage, in: Der Kunstwart 25 (August I9I2), 236-244, hier: $243 \mathrm{f}$.

20 Ludwig Geiger, Die deutsche Literatur und die Juden, Berlin I9Io, 5. 
alle von dem Streben erfüllt, ein neues Ghetto zu errichten, viel drückender als das alte, weil es ein selbstgeschaffenes ist, und weil es nicht diejenigen zurückdämmt, die durch Kleidung, Sprache und Gesittung sich von ihren Stadt- und Landgenossen unterscheiden, sondern weil es die durch die gesamte Kultur Geeinten, nur durch den Glauben Getrennten gänzlich voneinander zu scheiden sucht. ${ }^{2 \mathrm{I}}$ Sowohl gegen Antisemitismus wie auch gegen Zionismus hält deshalb Geiger, letztlich als Konsequenz aus der Emanzipation und der jüdischen Aufklärung, das Programm der deutsch-jüdischen Kultursymbiose. Der Politik der Exklusion und der Separation stellt er eine Politik der Inklusion und der Integration entgegen: "Nicht Antisemitismus und Zionismus [. . . k können mir die Überzeugung nicht [!] vernichten, daß der deutsche Gelehrte und Schriftsteller jüdischen Glaubens voll und ganz in Sprache und Gesinnung ein Deutscher ist, wohl berechtigt und befähigt, die Juden in der deutschen Literatur zu betrachten. $\aleph^{22}$ Die These, oder vielleicht besser: die Hoffnung dieser kulturellen Symbiose, als jüdischer Schriftsteller und Gelehrter "voll und ganz in Sprache und Gesinnung ein Deutscher zu sein", teilten, wie viele Porträts dieses Lexikons deutlich machen, zahlreiche jüdische Schriftsteller deutscher Sprache insbesondere im Wilhelminischen Kaiserreich ${ }^{23}$ und in der Weimarer Republik ${ }^{24}$. Die Partizipation an der deutschen Sprache, Literatur und Kultur schien ihnen der beste Garant einer liberalen, aufgeklärten jüdischen Moderne.

Diese Hoffnung wurde von der Geschichte wie kaum je eine andere zuvor enttäuscht, und die sich als »deutsche Staatsbürger jüdischen Glaubens "Verstehenden wurden mit Gewalt und gegen ihren Willen wieder zu Anderen, zu Juden erklärt - und als Juden vertrieben oder ermordet. Schon vor dieser Katastrophe, u. a. angesichts des zunehmenden Antisemitismus im letzten Drittel des I9. Jahrhunderts, wurde die Utopie der deutsch-jüdischen Symbiose von zionistischer Seite als der tragische Irrtum der bürgerlich-liberalen Ideologie des I9. Jahrhunderts scharf kritisiert. Die zionistische Kritik wandte sich gegen die Reduktion und Selbstauflösung jüdischer Kultur und Literatur, jüdischer Identität überhaupt. Tatsächlich argumentierte etwa Geiger, daß die literaturwissenschaftliche Beschreibung der deutsch-jüdischen Literatur letztlich darauf hinauslaufe, das zunehmende Verschwinden der jüdischen in der deutschen Kultur zu dokumentieren. Wenn Geiger I 897 einen »deutsch-jüdischen Roman« forderte, dann nicht etwa als Medium jüdischer Selbstvergewisserung, sondern als Dokumentationsorgan der voranschreitenden Assimilation: "Sollten unsere jüdischen Schriftsteller nicht geneigt sein, uns auch einmal einen deutsch-jüdischen Roman zu geben? Man möchte meinen, unsere Zeit fordere dringend dazu auf [...] das ganze sehnsüchtige Verlangen, sich ganz dem Volke zu vereinen, dem man durch Jahrhunderte lange Kultur und Gesittung angehört [...]; was gäbe das einem Dichter für einen Stoff: $\aleph^{25}$ Als ideologisch an

2 I Geiger, Die deutsche Literatur und die Juden, I I

22 Krojanker, Die Juden in der deutschen Literatur, I I.

23 Peter Gay, Die deutschen Juden in der Wilhelminischen Kultur, in: ders., Freud, Juden und andere Deutsche, München I989, i I 5-I 88 .

24 Michael Brenner, Die Jüdische Kultur in der Weimarer Republik, München 2000.

25 Ludwig Geiger, Zwei jüdische Erzählungen, in: Allgemeine Zeitung des Judentums 62 (I898), 366-368, hier: 368. Vgl. ders., Jüdische Romane, in: AZJ 68 (I904), 607-6Io. 
solcher Argumentation hat beispielsweise der Satiriker und Journalist Anton Kuh die "Selbstverleugnung" und den "Selbstbetrug", mehr noch: einen geradezu pathologischen "Selbsthaß « diagnostiziert. ${ }^{26}$ Gershom Scholem ging noch weiter, indem er als erklärter Zionist - nicht nur die deutsch-jüdische Symbiose als "Mythos" entlarvte, sondern auch die ihr entsprechende "Wissenschaft des Judentums" als eine fatale Strategie der Selbstliquidation kritisierte, deren offene Absicht es sei, dem Judentum »ein >ehrenvolles Begräbnis` zu bereiten« ${ }^{27}$

Diese Debatte zwischen Zionismus und Assimilation, deren Vielstimmigkeit die einzelnen Beiträge dieses Lexikons aufzeigen mögen, macht deutlich, daß das Konzept einer deutsch-jüdischen Literatur gerade innerhalb des Judentums zu einem zentralen Gegenstand seiner modernen Selbstbestimmung avancierte. Deutlich wird aber auch, daß eine aktuelle Rede von der deutsch-jüdischen Literatur genau diese Debatte analysieren müsste, jedoch ohne sich ihre auf unterschiedliche Weise apologetischen Positionen zu eigen $\mathrm{zu}$ machen, ohne also ihrerseits die deutsch-jüdische Literatur entweder apologetisch bzw. philosemitisch zur jüdischen $^{28}$ oder aber, aus einem historischen Mißverständnis heraus wie bei Gombrich, strikt zur deutschen Kultur zu rechnen.

Wie schwierig es ist, vor dem Horizont der verschiedenen historischen Interpretationen und Funktionalisierungen "jüdischer Kultur« und "jüdischer Literatur" angemessen von der deutsch-jüdischen Literatur zu sprechen, zeigt sich auch an den katastrophischen Bilanzziehungen nach I945. Wer nach I933/I945 von der deutschjüdischen Literatur sprechen wollte, befand sich gegenüber der breiten literarischen Produktion im Wilhelminischen Kaiserreich und der Weimarer Republik in einem völlig veränderten Dispositiv: Daß es jüdisches Schreiben in deutscher Sprache nicht mehr geben kann, aber auch, mit Blick auf die barbarisierte deutsche Sprache, nicht mehr geben dürfe, wurde im Ausmaß einer Katastrophe bewußt. Schon im Herbst I933 bilanzierte Arnold Zweig: "Die Sache der deutschen Juden [...] ist rund, abgeschlossen darstellbar«, wie er insbesondere für die Partizipation der Juden an der deutschen Literatur zeigte. ${ }^{29}$ Auch Siegmund Kaznelson, Leiter des Jüdischen Verlags bis in die fünfziger Jahre, konzipierte sein "Sammelwerk"Juden im deutschen Kulturbereich, das schon bei der Drucklegung im Jahr I935 verboten wurde, als eine "Schlussbilanz des deutschen Judentums«. Ähnlich formulierte es Robert Weltsch, als er Kaznelsons "Denkmal für die untergegangene deutsche Judenheit" I959 in erweiterter Form veröffentlichte: „Dieses Sammelwerk unternimmt den Versuch

26 Anton Kuh, Pogrom, in: Selbstwehr XII, Nr. 23 (23. Juni I9I8), 2.

27 Vgl. Gershom Scholem, Wider den Mythos vom deutsch-jüdischen Gespräch, in: Judaica 2, Frankfurt a. M. I970, 7-I I; Ders., Überlegungen zur Wissenschaft vom Judentum, in: Judaica 6, Frankfurt a. M. I995, 20 u. 29.

28 So argumentierte jüngst Dieter Lamping, indem er den Begriff der deutsch-jüdischen Literatur auf die spätestens I933 gescheiterte "Ideologie« der deutsch-jüdischen Symbiose zurückfuihrte und deshalb als verfehlt zurückwies. Statt von der deutsch-jüdischen müsse man vielmehr von der "jüdischen Literatur in deutscher Sprache" sprechen, um das hervorzuheben, "was jüdisch an ihr ist«. Dieter Lamping, Von Kafka bis Celan. Jüdischer Diskurs in der deutschen Literatur des 20. Jahrhunderts, Göttingen I998, I I.

29 Arnold Zweig, Bilanz der deutschen Judenheit 1933, Berliner Ausgabe, Berlin I998, 8. Zu den Juden in der deutschen Literatur vgl. I 82-I90. 
eines historischen Rückblicks auf den Anteil, den die deutschen Juden am wissenschaftlichen, künstlerischen, politischen, wirtschaftlichen, kurz: kulturellen und


Arthur Eloesser in seinem umfangreichen Beitrag zur deutsch-jüdischen Literatur in diesem Sammelband vor Augen führte, liest sich als eine Art defensive Apologie des jüdischen Beitrags zur deutschen Literatur angesichts seiner Verleumdung und Vernichtung. ${ }^{3 \mathrm{I}}$ Im selben Jahr brachte dies auch Kaznelson in seinem zweiten Sammelband Jüdisches Schicksal in deutschen Gedichten - eine abschließende Anthologie (I959) nachdrücklich zum Ausdruck, indem er das jüdische Schreiben förmlich testamentarisch vom deutschen Sprachraum verabschiedete: "Diese Sammlung wird als rabschließend bezeichnet, [...] weil nach menschlichem Ermessen die deutschsprachige Dichtung jüdischen Inhalts mit unsrer oder vielleicht der nächsten Generation zu Ende geht. So ist diese Anthologie ein Mahnruf, ein Vermächtnis [. . .] des untergegangenen deutschen Judentums an die Überlebenden. $\star^{32}$

Der neuere und aktuelle Diskurs über die deutsch-jüdische Literatur, Ausdruck eines Interesses nicht nur für die Geschichte, sondern auch für die Gegenwart und Lebendigkeit einer deutschsprachigen jüdischen Kultur, kann nicht mehr nur in tragischen Bilanzierungen und - daran anschließend - Musealisierungen des Judentums und seiner Kultur bestehen. Paradigmatisch für diesen Wandel ist der von Sander Gilman und Jack Zipes herausgegebene Yale Companion to Jewish Writing and Thought in German Culture, 1096-1996 (1997), der, wie die Jahresangabe »1096-1996« programmatisch deutlich macht, eine zwar veränderte, nicht aber gebrochene Tradition des jüdischen Schreibens in deutscher Sprache annimmt. ${ }^{33}$ Indem die Herausgeber, mit Rekurs auf Berthold Viertels Gedicht Der nicht mehr deutsch spricht (I94I), nicht nur eine "Jewish continuity in German language», sondern geradezu auch eine aktuelle "Jewish cultural renascence in Germany in den Blick rücken, relativieren sie die unter dem Eindruck der Katastrophe stehenden "Schlußbilanzen" des jüdischen Schreibens in deutscher Sprache und explizit auch Adornos berühmtes Diktum von I949/5I, "nach Auschwitz ein Gedicht zu schreiben, ist barbarisch ${ }^{34}{ }^{34}$

Wie nun läßt sich der Begriff der deutsch-jüdischen Literatur - vor dem Hintergrund seiner kontroversen historischen Interpretationen, der völkischen, der kulturzionistischen, der assimilatorischen und, wenn man will, der elegischen - gegen-

30 Juden im deutschen Kulturbereich. Ein Sammelwerk, hg. Siegmund Kaznelson, 2 Aufl., Berlin I959, XII.

3 I Arthur Eloesser, Literatur, in: Juden im deutschen Kulturbereich. Ein Sammelwerk, hg. Siegmund Kaznelson, 2 Aufl., Berlin I959, I-67.

32 Siegmund Kaznelson, Jüdisches Schicksal in deutschen Gedichten. Eine abschließende Anthologie, Berlin I959, I4. Vgl. ähnlich die von Karl Otten herausgegebenen Anthologien Prosa jüdischer Dichter, Stuttgart 1959, und Schofar. Lieder und Legenden jüdischer Dichter, Neuwied I962.

33 Sander L. Gilman, Jack Zipes (Hg.), Yale Companion to Jewish Writing and Thought in German Culture, 1096-1996, New Haven I997, xxiif.

34 Theodor W. Adorno, Kulturkritik und Gesellschaft (I95I), in: Gesammelte Schriften, hg. Rolf Tiedemann, Bd. Io.I, Frankfurt a. M. I977, 30. 
wärtig angemessen verwenden? Die Entwicklung, die sich am Yale Companion abzeichnet, kann nicht den Übergang zu einer "fröhlichen Wissenschaft" der deutsch-jüdischen Literatur bedeuten. Aus den historischen Bedingungen ergeben sich für eine aktuelle Verwendung des Konzeptes der deutsch-jüdischen Literatur vielmehr folgende Forderungen: Erstens muß die Gefahr der Ideologisierung immer mitbedacht und Polemik wie Apologie vermieden werden. Die Voraussetzung dazu ist - zweitens - das Wissen und Bewußtsein über die kontroversen historischen Interpretationen des Begriffs der deutsch-jüdischen Literatur. Vor diesem Horizont aber erweist sich - drittens - als die entscheidende Forderung an eine aktuelle Rede von der deutsch-jüdischen Literatur das Bewußtsein irreduzibler Mehrdeutigkeit dessen, was als deutsch-jüdische Literatur, als jüdische Identität überhaupt, gelten konnte und kann. Ideologie in der Rede über die deutschjüdische Literatur ist umgekehrt genau dort lokalisierbar, wo jüdische Identität eindeutig und totalisierend festzulegen unternommen wird. "Eindeutig" bedeutet entweder kollektiv, als handle es sich um eine soziologische Invariante, objektiv, als handle es sich um einen meßbaren und klassifizierbaren Gegenstand, oder essentialistisch, als handle es sich um eine metaphysische Qualität. Zweifel an der Möglichkeit der Rede von der jüdischen Kultur bzw. Literatur ist also genau da notwendig, wo sie auf potentiell ideologischen Zuweisungen beruht, die von der jüdischen Identität als einer Eindeutigkeit, einem Kollektiv oder einer Essenz ausgehen. Dagegen eröffnet umgekehrt der Blick auf die Mehrdeutigkeit dessen, was als "jüdisch" gelten kann, das Feld einer nicht-objektivierenden, nicht-totalisierenden und nicht-reduktiven Rede von der deutsch-jüdischen Literatur.

Diese Mehrdeutigkeit nun gelangt genau dann in den Blick, wenn nach dem Selbstverständnis und der Selbstbestimmung dessen, was deutsch-jüdische Literatur sei, gefragt wird. Ein Modell dieser Fragerichtung hat Stefan Zweig vorgeschlagen, als er von Martin Bubers Zeitschrift Der Jude den Einbezug auch der Literatur forderte. Zweigs Vorschlag ist signifikant: Er regte an, daß alle deutsch-jüdischen Autoren in Bubers Zeitschrift eine Stellungnahme zu ihrem Verständnis des Judentums formulierten: "Was die Stellung zum Judentum betrifft - wäre es nicht die Aufgabe gerade ihrer Revue in einer Art großzügigen Rundfrage von jedem deutschen Autor jüdischen Ursprungs ein Bekenntnis seiner Stellung zu verlangen? Es wäre für jeden vielleicht Entlastung, gezwungen zu sein, sich selbst gegenüber Farbe zu bekennen und andererseits ein ungeheures Dokument für spätere Zeiten. «5 $^{35}$ Das entscheidende Moment in Zweigs Vorschlag, das auch und gerade für eine aktuelle Rede von der deutsch-jüdischen Literatur von Bedeutung ist, ist die Selbstbestimmung: sie ersetzt verallgemeinernde Zuschreibungen durch selbstreflexive Standortbestimmungen singulärer Schreibakte. Es ist demnach nicht die Aufgabe der Literaturwissenschaft, selbst festzulegen und $\mathrm{zu}$ bestimmen, was deutsch-jüdische Literatur sei, welche Autoren zu ihrer Geschichte und welche Texte zu ihrem Korpus gehören. Es geht nicht darum, Normen und Kriterien wie Herkunft, Substanz, Stoffe, Motive, Sprache, Stil vorzugeben, um zu einer ein-

35 Martin Buber, Briefwechsel aus sieben Jahrzehnten, hg. Grete Schaeder, 3 Bde., Heidelberg I972, Bd. I, 43I. 
deutigen Definition der deutsch-jüdischen Literatur zu gelangen; dies wäre erneut objektivierend und totalisierend. Die Aufgabe einer wissenschaftlichen Beschreibung wird vielmehr darin bestehen, ihrerseits literarische Selbstbestimmungsdiskurse zum Gegenstand $\mathrm{zu}$ machen und $\mathrm{zu}$ fragen, mit welchen argumentativen Verfahren in den verschiedenen historischen Debatten, letztlich aber in jedem einzelnen Schreibakt, in jedem einzelnen Text, der irreduzibel vieldeutige interkulturelle Raum der deutsch-jüdischen Literatur konstruiert und interpretiert wird.

Das Medium nun, das diese Perspektive auf die deutsch-jüdische Literatur angemessen darzustellen vermag, ist das Lexikon. Die lexikographische Fragmentierung eines literaturgeschichtlichen Zusammenhangs in einzelne Profile kultureller Schreiborte ist förmlich die schreibtechnische Antwort auf die Frage nach der Selbstpositionierung der einzelnen Autoren im Schreibraum der deutsch-jüdischen Interkulturalität. In dieser fragmentierten Gestalt wollen die Porträts sichtbar machen, daß und wie die deutschsprachigen jüdischen Autorinnen und Autoren in ihren literarischen wie autobiographischen Schriften den Diskurs über den kulturellen Schreibort der deutschsprachigen Juden formiert und an ihm teilgenommen haben. Folglich erscheinen die einzelnen Autorinnen und Autoren in diesem interkulturellen Feld weniger, weil sie jüdischer Herkunft sind oder weil sie jüdische Stoffe thematisieren, sondern um zu fragen, wie sie mit ihrem Schreiben auf je eigene und unterschiedliche Weise an der jüdischen Selbstbestimmung der Moderne partizipiert haben. Dabei können die allgemeineren Positionen der vielgestaltigen Debatten seit der Aufklärung nur den weitesten Rahmen abstecken. Das gilt etwa für die Debatten über verschiedene Formen jüdischer religiöser Identität wie (Neo-) Orthodoxie, Chassidismus, Reformjudentum und das Problem der Konversion; für die politischen Debatten über den Europabegriff, Deutschnationalismus, Zionismus, Bundismus und sozialistische Internationale; für die kulturellen Debatten über Assimilation und Akkulturation, über die kulturelle Differenz zwischen Westjudentum und Ostjudentum, über die Bedeutung einer "jüdischen" Kultur, Literatur und Sprache überhaupt; schließlich für Debatten über historische Ereignisse und Erfahrungen wie Emanzipation und Bürgerrecht, Antisemitismus und Verfolgung, Exil und Shoah, das Verhältnis zum Staat Israel und zu Deutschland nach I945, etc. Die Singularitäten der Interpretationen manifestieren sich also nicht in diesen Topoi jüdischer Identität in der Moderne selbst, sondern werden erst in den je eigenen Stellungnahmen zu und in der Art und Weise der Teilnahme an jenen Debatten manifest. Die Möglichkeiten reichen von aktiver und engagierter Stellungnahme (etwa bei Arnold Zweig oder Max Brod) über eine mehr oder weniger indirekte, parabolisch verschlüsselte und allusiv verfahrende Zitierweise, die eine eindeutige Positionsbestimmung gerade vermeidet (etwa bei Franz Kafka), bis hin zu expliziten Verweigerungen der Teilnahme an einem jüdischen Diskurs, sei es in einer polemischen Auseinandersetzung mit den Möglichkeiten jüdischer Identität in der Moderne (z.B. bei Karl Kraus oder Anton Kuh), oder aber durch Konversion (z.B. Heine und Börne) oder einen demonstrativen Austritt (etwa bei Kurt Tucholsky). Insbesondere an letzterem wird deutlich, daß es nicht darum gehen kann, einzig nach den >positiven Stellungnahmen zum Judentum, nach Bejahungen und Identifikationen oder gar nach apologetischen Literarisierungen zu fragen. Die 
Frage nach der Interpretation und Selbstbestimmung des Judentums muß vielmehr in einer möglichst komplexen Form gestellt werden, die nicht nur Identifikationen und explizite Stellungnahmen zum Judentum wahrnehmbar macht, sondern auch und gerade Traditionsbrüche, Fremdheitserfahrungen, Differenzen, Distanzen und parabolische Transformationen. Die jüdische Moderne seit der Aufklärung, der die deutschschreibenden jüdischen Autoren angehören, besteht gerade in einem Bruch mit Traditionalismus und Konformismus. Sie erweist sich immer schon als eine Vielfalt verschiedener Formen der Transformation und Transgression von jüdischen Traditionen, von Tradition überhaupt.

Damit ist auch das historische Korpus des vorliegenden Lexikons angesprochen, das nun, nach der begriffsgeschichtlichen Klärung, zumindest in seinen Grundzügen umrissen werden soll. Wenn es nämlich so etwas wie einen Anfang der deutschjüdischen Literatur im I8. Jahrhundert gibt, dann ist es eben diese Transgression der hebräischen Tradition bzw. die Öffnung des jüdischen Schreibens hin zur deutschen Sprache und zu einem säkularen und damit auch zu einem literarischen Schreiben. Moses Mendelssohns deutsche Übersetzung der Bibel (I78I), seine ästhetischen und literaturkritischen Schriften, aber auch seine Freundschaft mit Lessing, sind initial und beispielhaft für diesen Schritt. Die weitere Geschichte dieses Schreibens - das freilich nicht an Deutschland gebunden ist, sondern seinen Raum auch in den deutschsprachigen Literaturlandschaften Osteuropas hat, insbesondere aber im Österreich der weiten Grenzen der k. und k. Monarchie, das Prag ebenso wie Galizien einschloß, ${ }^{36}$ spätestens seit 1933 zudem in allen europäischen und außereuropäischen Schreiborten des Exils von Palästina bis Mexiko - läßt sich hier nur in einigen seiner Stationen vor Augen halten. Die kulturpolitischen Kategorien der Aufklärung, die von jüdischer wie von nichtjüdischer Seite her die Partizipation der Juden am kulturellen Leben Europas möglich machten - u.a. Akkulturation und Emanzipation -, waren auch die Voraussetzungen der Berliner jüdischen LiteraturSalons, geführt von Schriftstellerinnen wie Henriette Herz, Rahel Varnhagen und Fanny Lewald, ${ }^{37}$ und bestimmen auch das Schreiben jüdischer Schriftsteller aus dem osteuropäischen Raum wie Salomon Maimon. Was Kant für Maimon bedeutete, war Goethe für Rahel Varnhagen: Inbegriff und Garant einer modernen, aufgeklärten und humanistischen europäischen Kultur, die auch Raum für eine moderne jüdische Identität bot.

Diese Integration war dennoch meist an eine weitgehende Aufgabe jüdischer Identität, auch der konfessionellen, gebunden. Unter anderen waren auch Heinrich Heine und Ludwig Börne dieser Dialektik der Aufklärung ${ }^{38}$ ausgesetzt, wenn sie ihre Konversion, nunmehr angesichts eines zunehmenden deutschen Nationalismus und auch Antisemitismus seit der späteren Romantik, als »Entréebillet zur europäi-

36 Vgl. Harry Zohn, Österreichische Juden in der Literatur. Ein bio-bibliographisches Lexikon, Tel Aviv 1969; ders., Wiener Juden in der deutschen Literatur, Tel Aviv 1964.

37 Vgl. Deborah Hertz, Die jüdischen Salons im alten Berlin 1780-1806, München 1995.

38 Vgl. Hannah Arendt, Aufklärung und Judenfrage, in: dies., Die verborgene Tradition. Acht Essays, Frankfurt a. M. 1976, I08-I26. 
schen Kultur« verstanden. Als Kritiker der deutschen Romantik und am revolutionären Frankreich orientiert inaugurierten Heine und Börne eine Feuilletonkultur, die für zahlreiche, noch so unterschiedliche jüdische Intellektuelle, von Moritz Gottlieb Saphir und Daniel Spitzer im I9. Jahrhundert bis zu Theodor Herzl, Max Nordau, Felix Salten, Peter Altenberg, Alfred Polgar, Karl Kraus und Anton Kuh im ersten Drittel des 20. Jahrhunderts das entscheidende Medium des Schreibens - auf verschiedenste Weise auch einer jüdischen Selbstreflexion - war. Zu den Formen des jüdisches Schreibens im I9. Jahrhundert zählte neben dem Feuilleton auch das Drama, für das beispielhaft Michael Beers Der Paria (1826), Karl Isidor Becks Saul (I840) oder Salomon Hermann Mosenthals Deborah (I850) genannt seien, ebenso auch die sogenannte "Ghettoliteratur", Schilderungen jüdischen Lebens in osteuropäischen Städtchen. ${ }^{39}$ Anders als Berthold Auerbachs Schwarzwälder Dorfgeschichten, mit denen er sich nach den beiden Romanen über Spinoza und Ephraim Moses Kuh von jüdischen Themen fast gänzlich abgewandt hat, wurde die Ghettoliteratur, deren wichtigste Autoren Leopold Kompert und Karl Emil Franzos waren, von den bürgerlich-liberalen jüdischen Lesern zurückgewiesen. Seit dem Ende des I9. Jahrhunderts jedoch fand sie, auch vor dem Hintergrund der kulturzionistischen Entdeckung des Ostjudentums als eines ursprünglichen und lebendigen Judentums und zusammen mit Übersetzungen chassidischer und moderner jiddischer Literatur (z. B. Martin Buber, Alexander Eliasberg) große Beachtung.

Der aufkommende Zionismus, aber auch der zunehmende Antisemitismus um I900 ging mit einer Polarisierung der deutschsprachigen jüdischen Literatur einher: die ältere Position des aufgeklärten bürgerlich-liberalen Judentums, wonach die deutsch-jüdische Literatur als das hervorragende Medium der Akkulturation galt, tendierte seit den Gründerjahren, insbesondere aber vor dem Hintergrund des Ersten Weltkriegs, teilweise zu einer nahezu bedingungslosen Loyalität gegenüber Deutschland bzw. Österreich. Dagegen formierte sich eine zionistisch orientierte deutsch-jüdische Literatur, deren Movens die »Renaissance des Judentums» war. Herzls Roman Altneuland (I9O2) ist freichlich mehr als nur Beispiel dieser Literatur, an der mit Überzeugung u.a. Berthold Feiwel und Adolph Donath, aber auch Richard Beer-Hofmann oder Max Brod schrieben. Zahlreiche deutsch-jüdische Schriftsteller des Kaiserreichs und mehr noch der Weimarer Republik reflektierten diesen Konflikt zwischen den beiden kulturellen Identitäten, der deutschen und der jüdischen, so beispielsweise Ludwig Jacobowski, etwa in seinem Roman Werther, der Jude (I 892), oder Jakob Wassermann, von seinem Roman Die Juden von Zirndorf (1897) bis zu seinem Essay Mein Weg als Deutscher und Jude (I92I), oder Arthur Schnitzler, etwa in seinem Roman Der Weg ins Freie (I908) oder seinem Drama Professor Bernhardi (1912). Zahlreiche jüdische Autoren, nicht wenige Vertreter des Expressionismus, standen, oft als erklärte Pazifisten, zwischen oder auch jenseits der Opposition von Akkulturation und Zionismus, wenn sie beide Positionen gleichermaßen kritisierten und dagegen eine aufgeklärt-kosmopolitische oder auch sozialistisch-internationale Europaidee favorisierten, in der das Judentum in unter-

39 Vgl. Gabriele von Glasenapp, Aus der Judengasse. Zur Entstehung und Ausprägung deutschsprachiger Ghettoliteratur im 19. Jahrhundert, Tübingen 1996. 
schiedlicher Weise integriert war, so etwa Ernst Toller, Alfred Döblin, Joseph Roth, Stefan Zweig, Else Lasker-Schüler, Franz Werfel, Yvan Goll, Lion Feuchtwanger oder Alfred Wolfenstein, der in seinen Essays, z.B. in Jüdisches Wesen und neue Dichtung (1922), den Dichter geradezu als Inbegriff jüdischer Diasporaexistenz verstand.

Mit dem Aufstieg des Nationalsozialismus und der Machtergreifung I933 in Deutschland und dem österreichischen Anschluß I938 wurde der Antisemitismus zu einem akuten Problemfeld für einen jeden mit seinem Schreiben öffentlich auftretenden deutsch-jüdischen Autor, deutlich etwa bei Lion Feuchtwanger und Arnold Zweig. Vor allem für diejenigen, die noch nach 1938 in Deutschland verweilten, wurde das Schreiben zu einem Ansturm gegen das Äußerste, so etwa bei Gertrud Kolmar, Paul Kornfeld, Georg Hermann, Erich Mühsam oder Arno Nadel, einem Äußersten, das nicht wenige deutsch-jüdische Autoren, auch im Exil, in den Selbstmord getrieben hatte (Egon Friedell, Ernst Toller, Kurt Tucholsky, Ernst Weiss, Alfred Wolfenstein, Stefan Zweig u.a.). Was an deutsch-jüdischer Literatur nach 33/38 noch möglich war, wurde zum größten Teil "Exilliteratur«: Das Exil, vor allem in den USA und in Palästina, aber auch in England, Frankreich und in zahlreichen anderen Ländern wurde - erneut - zum entscheidenden Dispositiv des jüdischen Schreibens in deutscher Sprache. ${ }^{40}$

Nach 1945 wog die Tatsache schwer, daß jüdisches Schreiben in deutscher Sprache einen katastrophalen Bruch erlitten hatte; auch wo Deutsch zu schreiben noch möglich gewesen wäre, schien dies, mit Blick auf die barbarisierte deutsche Kultur, nicht mehr vertretbar. Jetzt, ein halbes Jahrhundert nach diesem Bruch, wird immer deutlicher, daß die Geschichte der deutsch-jüdischen Literatur nie wirklich "abgeschlossen" war, seit I945 aber, kaum anders heute noch, im Zeichen erlittener Gewalt steht. Dies gilt für die Versuche, das Unsägliche in einer zugleich moralischen und ästhetischen Gratwanderung zur Sprache zu bringen (z.B. Paul Celan, Nelly Sachs, Rose Ausländer, Wolfgang Hildesheimer, Jurek Becker, Ruth Klüger). Es gilt aber auch für das Schreiben einer jüngeren, "zweiten Generation", insbesondere da, wo sie das Nachkriegsdeutschland und den jungen israelischen Staat zum Thema macht (z. B. Barbara Honigmann, Robert Menasse, Doron Rabinovici, Maxim Biller, Gila Lustiger). Das Schreiben der deutsch-jüdischen Literatur kann folglich nicht mehr als beendet verstanden werden; es wird, wenn auch ganz anders als vor I933 und kaum anders als unter dem Eindruck erlittener Gewalt, fortgeschrieben. Auch um dies zu zeigen, sind in diesem Lexikon gegenwärtig schreibende Autorinnen und Autoren mitaufgenommen.

Dieser kurze historische Überblick soll und kann nicht ersetzen, worauf in diesem Lexikon bewußt verzichtet wird: die Konstruktion eines literaturgeschichtlichen Zusammenhangs. Wie schon mit der Fokussierung auf den Diskurs der Selbstbestimmung jede Einheitlichkeit im Begriff des Judentums und der deutschjüdischen Literatur vermieden wird, soll auch auf jede Form von Linearität in der

40 Vgl. Itta Shedletzky/Hans Otto Horch (Hg.), Deutsch-jüdische Exil- und Emigrationsliteratur im 20. Jahrhundert, Tübingen I993. 
Geschichte verzichtet werden. Die einzelnen Porträts können allenfalls als Bausteine einer deutsch-jüdischen Literaturgeschichte gelesen werden, ohne jedoch selbst eine solche zu konstituieren. ${ }^{4 \mathrm{I}}$ Das vorliegende Lexikon hypostasiert in seinen Einzeldarstellungen keine gesonderte Geschichte, sondern bildet gleichsam ein Mosaik aus Porträts, die am Leitfaden von Lebensläufen und literarischen wie autobiographischen Texten die mehr oder weniger expliziten Formationen und Perzeptionen des vielstimmigen Diskurses über das Judentum sichtbar machen. Deutsch-jüdische Literatur ist in diesem präzisen Sinne eine historisch und politisch-geographisch deterritorialisierte Literatur, eine Literatur zwischen Ereignissen, Nationen und Politiken. Deshalb will das Lexikon statt begrifflicher Totalisierung der deutschjüdischen Literatur die irreduzible Pluralität singulärer Interpretationsakte sichtbar machen, statt historiographischer Linearisierung Momentaufnahmen geben, statt geographisch-nationalliterarischer Territorialisierung die Atomisierung und "Exilierung« in je aktuelle Schreiborte vor Augen führen.

Wenn das Lexikon auf diese Weise Zusammenhänge und Ganzheiten vermeidet, dann gilt dies schließlich auch für das Korpus der Artikel: Vollständigkeit kann und will es nicht anstreben. Aus pragmatischen Gründen kann es dies nicht, denn ein annähernd vollständiges, also enzyklopädisches Lexikon der deutsch-jüdischen Literatur müsste um ein mehrfaches umfangreicher sein und eine andere historische Forschungslage zur Voraussetzung haben; aus konzeptuellen Gründen will es dies nicht, denn es gibt schlechterdings keine vertretbaren Auswahlkriterien, die so etwas wie Vollständigkeit in der Bestandsaufnahme jüdischer Autoren deutscher Sprache erlaubten. Es wird deshalb nicht zu vermeiden sein, daß einzelne Namen vermißt werden, die bisher, wenn überhaupt, nur in rein faktographischen Nachschlagewerken aufgeführt sind. ${ }^{42}$ Dennoch bedeutet dies gewiß nicht, daß das vorliegende Lexikon nur die bekanntesten Namen berücksichtigt. Die Frage nach der jüdischen Selbstbestimmung bzw. der Partizipation an jüdischen Selbstbestimmungsdiskursen der Moderne gilt gerade auch einer großen Anzahl von wenig bekannten Namen, Namen also, die im Zuge der Katastrophe des 20. Jahrhunderts zu denen zählen, die

4I Zu literarhistorischen Beschreibungen der deutsch-jüdischen Literatur vgl. Hans Otto Horch, Deutsch-jüdische Literatur I. Vom 18. Jahrhundert bis zu Heinrich Heine, Studienbrief Fern-Universität Hagen I995; ders., Deutsch-jüdische Literatur II. Von Heinrich Heine bis zur Wiener Moderne, Studienbrief Fern-Universität Hagen I998; Hans Schütz, Juden in der deutschen Literatur: Eine deutsch-jüdische Literaturgeschichte im Überblick, München I992.

42 Eine etwas größere Auswahl kurzer bio-bibliographischer Artikel auf älterem Stand enthält Desider Stern, Werke jüdischer Autoren deutscher Sprache. Eine Bio-Bibliographie, 3. Aufl., Wien I970. Die größte Auswahl bietet - allerdings als reine Namenliste - die Bibliographia Judaica. Verzeichnis jüdischer Autoren deutscher Sprache, 3 Bde. und ein Ergänzungsband, bearbeitet v. Renate Heuer, Frankfurt a.M. I982-I995. Eine Auswahl dieser Liste erscheint - hier wiederum nicht in ausformulierten Lexikoneinträgen, sondern in bio-bibliographischen Stichworten - im mehrbändigen Lexikon deutsch-jüdischer Autoren. Archiv Bibliographia Judaica, redaktionelle Leitung: Renate Heuer, München u.a. I992ff. Der Begriff des "Autors « ist in diesen beiden Lexika sehr weit gefaßt und meint neben belletristischen auch philosophische und wissenschaftliche Autoren aller Disziplinen. Zu weiteren Lexika vgl. die Bibliographie. 
nicht nur "verboten" und "verbrannt", sondern auch "vergessen" und "verkannt» waren. ${ }^{43}$

Dabei wird sich zeigen, daß Bekanntheit ein Maß für "Bedeutung" nicht sein kann. Im Gegenteil: die Aufmerksamkeit - dies das eigentliche Movens dieses Vorhabens - gilt gerade einem Unbekannten, einem Unbekannten nicht nur bei vergessenen, sondern auch bei bekannten Autoren, einem Unbekannten nämlich, das mit ihrem Judentum aufs engste zusammenhängt, einem Unbekannten damit unserer eigenen europäischen Kultur. In dem $\mathrm{Maß}$ aber, wie das Lexikon in dieses Unbekannte vordringt, mag es dazu beitragen, das Feld der deutschen Literatur zu öffnen und mit einem im 20. Jahrhundert gewaltsam aus ihrem Bereich ausgeschlossenen Schreiben neu bekanntzumachen: dem Schreiben der deutsch-jüdischen Literatur.

Dieses Lexikon ist von Anfang an auch ein Gemeinschaftswerk; erst im Vollzug eines unverzichtbaren Austauschs und Gesprächs mit jedem einzelnen der Mitarbeiter hat es seine vorliegende Gestalt angenommen. Besonderer Dank gilt darüber hinaus Detlef Kremer und Claudia Lieb (Institut für deutsche Philologie II, Münster) sowie Monika Brand (Köln) und Ursula Steck (Köln), die auf je eigene Weise die Realisation dieses Lexikons mitermöglichten. Dank gilt schließlich auch Gabriele Aichele (Bildredaktion) und insbesondere Oliver Schütze vom Metzler-Verlag, der das Vorhaben in seiner Entstehungszeit mit vielfältigem Rat begleitet hat.

Köln/Münster im Dezember I999

Andreas B. Kilcher

43 Vgl. die beiden Lexika Verboten und Verbrannt. Deutsche Literatur - 12 Jahre unterdrückt, hg. Richard Drews/Alfred Kantorowicz, Berlin I947; Hans Schütz, Ein Deutscher bin ich einst gewesen. Vergessene und verkannte Autoren des 20. Jahrhunderts, München I988. 